\title{
Dimension with Heterogeneous Module
}

National Cancer Institute

\section{Source}

National Cancer Institute. Dimension with Heterogeneous Module. NCI Thesaurus. Code C113738.

A proprietary integ rated chemistry system for high-throug hput integration of chemistry and immunoassay testing manufactured by Siemens. 